Norris, C. E., \& Colman, A. M. (1996). Context effects of radio programming on cognitive processing of embedded advertisements. Applied Cognitive Psychology, 10, 473-486.

\title{
Context Effects of Radio Programming on Cognitive Processing of Embedded Advertisements
}

\author{
CLAIRE E. NORRIS \\ De Montfort University \\ and \\ ANDREW M. COLMAN \\ Leicester University
}

\begin{abstract}
SUMMARY
This experiment tested the hypothesis that the involvement, entertainment, and enjoyment properties of radio programmes would be related to memory for the accompanying advertisements and attitudes towards them. Ninety-three subjects listened to one of three radio programmes (a phone-in, a chart show, or a nostalgia programme) in which were embedded a set of four unfamiliar advertisements. Subjects' ratings of the programmes as involving, entertaining, and enjoyable were positively correlated with subsequent ratings of the advertised brands and purchasing intentions. Analyses of variance indicated significant differences in memory for the advertisements and programme ratings: the phone-in programme context, which was rated significantly less interesting, enjoyable and entertaining, and more boring and humorous than the nostalgia radio programme context, produced significantly lower memory for the advertisements. These results provide substantial evidence for context effects operating in the radio medium.
\end{abstract}

This study focuses on the relationship between radio programme material and the cognitive processing of advertisements that accompany it. Radio advertisements are not heard in isolation, but generally appear within the context of either programmes or other non-programming material, such as other advertisements or continuity announcements. Programming context material varies in many ways, and the various contexts may differentially influence how the advertising messages within them are attended to, cognitively processed, and therefore remembered. People listen to the radio for the programmes rather than the advertisements, and thus it is possible that their reactions to and feelings about the programmes may influence their reactions to advertisements inserted within them. In other words the effectiveness of the advertising message may partly depend on the context in which it is embedded.

Within the literature on television advertising, the programme context in which an advertisement appears has often been hypothesized to influence the cognitive processing of advertisements (e.g., Axelrod, 1963; Barclay, Doub \& McMurtrey, 1965; Bryant \& Comisky 1978; Clancy \& Kweskin, 1971; Horn \& McEwan 1987; Kamins, Marks \& Skinner, 1991; Kennedy, 1971; Krugman, 1983; Lloyd \& Clancy, 1991; Norris \& Colman, 1993, 1994a, 1994b; Park \& McClung, 1986; Schumann, 1986; Schwerin \& Newell 1981; Soldow \& Principe, 1981; Thorson \& Reeves, 1986; Thorson, Reeves \& Schleuder 1985). Context effects in the print medium have also occasionally been studied (Norris \& Colman, 1992; Yi, 1990a, 1990b), but context effects in the medium of radio broadcasting do not appear to have been investigated. In fact, radio advertising has often been investigated in isolation of its programming context. Several studies have concentrated on a comparison of advertisement 
effectiveness between the three major mass media: television, radio, and print (e.g., Furnham, Benson \& Gunter, 1987; Klein, 1981; Warshaw, 1978; Williams, Paul \& Ogilvie, 1957). Other studies have exposed subjects to radio advertisements within the natural programming environment but have not investigated the relationship between that environment and the advertisement effectiveness (e.g., Duncan \& Nelson, 1985; Gelb \& Zinkhan, 1985, 1986).

In a study focusing on car radio listening, Abernethy (1991) acknowledged the dependent relationship between the radio programme and the embedded advertisements. Subjects within his study were 101 19-24 year olds, who were considered to be a primary audience for many radio stations. Abernethy recorded these subjects' exposure to radio stations and the embedded advertisements and observed that subjects would switch radio stations to specifically avoid the advertisements. This suggests that what is needed from an advertiser's point of view is an investigation into the types of programmes that would sufficiently involve listeners so that they do not wish to switch channels when the advertisements appear. Most studies investigating radio advertisements have not, however, considered the relationship between listeners' responses to programmes and advertisement effectiveness. The study reported in this article therefore aimed at investigating the possible relationship between reactions to radio programmes and memory for and attitudes towards accompanying advertisements.

The numerous studies investigating television context effects, which must inevitably provide a background for this study, have generated inconsistent results (for a review, see Norris \& Colman, 1993). Some have reported a positive relationship between predictor variables and advertisement effectiveness, with highly rated contexts associated with high advertisement effectiveness, while others have reported a negative or inverse relationship. Various theories have been put forward to explain these apparently conflicting findings. Where a negative relationship is reported, some kind of reference is often made to an information processing model, in particular to the existence of a limited capacity cognitive processing system. Attention has focused on the effects of cognitive processing demands on attention or the resulting interference from competing stimuli. Conversely, where a positive relationship is reported, reference is generally made to some sort of facilitative priming or carry-over effect from the programme to the advertisements causing the audience to be more receptive to advertisements while viewing to highly rated programmes. These contrasting theories are reflected in Schumann and Thorson's (1990) classificatory model of context effects. The study reported in this article poses the question of whether effects of contexts on advertisement effectiveness in the radio medium are positive or negative. In particular, this study examines the hypothesis that subjects' ratings of radio programmes as involving, entertaining, or enjoyable are related to advertisement effectiveness.

As in the Abernethy (1991) radio study, subjects were chosen to reflect an age group that is considered to be a primary audience for the radio programmes used in the study. The advertisements and programmes were selected from a large pool, and steps were taken to ensure that the programming materials and advertised brands were entirely unknown by all subjects, thus controlling for the problem of prior exposure which vitiates much of the research involving recall and recognition measures in this area. The study reported below included sophisticated measures of recall and recognition of advertisements, product types, and brand names, and also measures of attitudes towards the advertisements and intentions to buy the products. Subjects listened to one of three radio programmes interspersed with four advertisements and 
were afterwards asked to respond to a series of questionnaires. A laboratory study was used in preference to a naturalistic survey because it provided an opportunity for strict control of the context variables and of the many extraneous factors that might influence advertisement effectiveness. This was considered important because the inconsistencies in the television context literature have often been attributed to methodological problems (Norris \& Colman, 1993, 1994a, 1994b; Schumann, 1986).

\section{Subjects}

\section{METHOD}

The subjects who participated in this experiment were 93 undergraduate students (43 men and 50 women) at the University of Leicester, aged between 18 and 34 years (mean $=20.27, S D=3.34$ ), who received $£ 2.50$ for their participation. Subjects were randomly assigned to one of three treatment conditions associated with three different context programmes, with the limitation that each treatment condition had an equal number of subjects.

\section{Design and materials}

\section{Radio programmes}

The three context programmes were all selected from radio stations outside the Leicestershire broadcasting region. This enabled English-language material to be used that the subjects were unlikely to have heard before. The programmes, chosen to create three sharply contrasting contexts, were as follows:

Condition 1: Phone-In (22 minutes and 35 seconds). This was a typical phone-in programme in which members of the public talk to a disc jockey about various issues. The topics featured included exercise addiction, junk mail, statistics, and opinion polls.

Condition 2: European Chart Show (20 minutes and 40 seconds). This featured popular music hits from artists such as Janet Jackson, Duran Duran, Karen Wheeler, The Farm, and other music appearing in a European chart show of November 1990.

Condition 3: Nostalgia programme: 'Rock Decades' (20 minutes and 55 seconds). This was a nostalgic programme featuring music and simulated news excerpts from 1967. Hits such as Lets Spend the Night Together (Rolling Stones), Hi Ho Silver Lining (Jeff Beck), R.E.S.P.E.C.T. (Aretha Franklin), and All You Need is Love (Beatles) were featured.

\section{Advertisements}

Two advertisement breaks (55 seconds and 41 seconds respectively), each containing two advertisements, were inserted within the radio programmes. Identical advertisement breaks were used in all three treatment conditions. Two of the advertisements (Fit for the Queen exhibition and Cactus warehouse) were taken from radio stations outside of the Leicestershire broadcasting region again to ensure that the advertisements and brand names were unfamiliar to subjects. It was decided to use as the other two advertisements the soundtrack from Australian television advertisements. This was necessitated by a dearth of radio advertisements (during an economic recession) for products with unfamiliar brand names. The two Australian advertisements were selected from a total sample of 41 advertisements recorded off air from Australian and South African Television channels; the advertisements finally selected were for Winner ice cream bar and IXL jam. To ensure that these advertisements were suitable for radio, they were pretested in a pilot study to confirm that they sounded like radio advertisements, and in particular that the product, brand 
name, and other details were fully comprehensible from the soundtrack. A second pilot study also confirmed that the brand names were all unfamiliar to British listeners.

The order of the four advertisements in the two advertising breaks was determined randomly by blind lottery. The first commercial break contained the advertisements for the ice cream bar and the exhibition and was edited in at a mean of 6.05 minutes $(S D=$ 0.06) after the beginning of the programme. The second break contained the advertisements for the jam and the warehouse and appeared a mean time of 15.11 minutes $(S D=0.15)$ after the beginning of the programme. A brief description of the advertisements is as follows.

(a) Winner ice cream bar: A backing rock song begins, 'No place to hide, this town is on fire...' . A male voice-over is heard: 'It takes a cool bar to stay up in a hot world.' We are told that only Winner, with its creamy ice cream packed with delicious crunchy nuts topped with thick caramel and milk chocolate, can keep you on the 'up and up'. A voice-over ends the advertisement with: 'Go with the cool energy of Winner, the up bar that keeps you cool'.

(b) Fit For The Queen exhibition: A fanfare begins the advertisement and a male voice-over says, 'Over the years many different companies have made a big noise in the business world'. Several different sound effects can be heard representing different products, such as cows mooing and marching feet. A voice-over ends off with: 'To find out which companies are behind these diverse products visit Fit for the Queen, a Design Council Exhibition celebrating 25 years of the Queen's Awards for export and Technology'.

(c) IXL jam: Easy-listening piano/guitar music is heard as a male voice-over says, 'For almost 100 years generations of Australian families have loved the taste of IXL jam'. We are told that IXL uses only quality fruit that 'ensures our jam truly excels'. The voice-over states, 'That's why you can tell when it's IXL'.

(d) Cactus warehouse: Rock music is heard with a female voice singing, 'It's a world of value and choice when you open the door to cactus'. A male voice-over says, 'Register your company at the huge Cactus trade warehouse and reap the benefits'. We are told that there are top brand names at trade prices at Cactus warehouse.

The reference to 'Australian families' in (c) above did not make the advertisement seem unreal to the subjects, as confirmed by the pilot study mentioned earlier. It is not unusual for marketing of produce from Commonwealth countries to refer to the popularity of those products in their countries of origin.

\section{Questionnaires}

Several questionnaires were used to measure the subjects' perceptions of the programmes and advertisements and their recall and recognition of the advertisements. After supplying details of their sex and age, subjects were asked if they had ever heard the radio programme prior to the experiment (none of them had done so). Subjects then responded to the following six questionnaires in the order shown:

(a) Programme ratings. Subjects responded to a counterbalanced set of 21 7-point rating scales relating to the radio programmes they had just listened to as regards to their entertainment, enjoyment, and involvement properties. These rating scales were based on similar scales from earlier investigations in which programme-induced involvement, entertainment, and enjoyment were the predictor variables (Bryant \& Comisky, 1978; Norris \& Colman, 1992, 1993; Park \& McClung, 1986; Soldow \& 
Principe. 1981). They were also derived from empirical definitions of each of the three terms (involvement, entertainment, and enjoyment) obtained from a cluster analysis of a very large number of candidate items including all those that have been used in previous published research (Norris \& Colman, 1994b).

(b-e) Measures of recall and recognition. Subjects' recall and recognition of the advertisements were measured with the following four measures adapted from those used in previous context research (Bryant \& Comisky, 1978; Heflin \& Haygood, 1985; Kennedy, 1971; Murphy, Cunningham \& Wilcox, 1979; Norris \& Colman, 1992, 1993; Schumann, 1986; Soldow \& Principe, 1981; Thorson, Friestad \& Zhao, 1987).

(b) Free recall: subjects were asked to write down as much as they could remember about the advertisements including brand name, product and details of the advertisement.

(c) Recognition of products: subjects were asked to recognize the four product types from among a total of 33 randomly organized products types also commonly advertised on radio; the four target product types appeared randomly within the other 29.

(d) Cued recall of brand names: the four product types relating to the target advertisements were given and subjects were asked to recall the corresponding brand name.

(e) recognition of brand names: each brand name was randomly placed beneath the relevant product type with five other possible brand names. The subject's task was to circle the appropriate brand name. For authenticity, the non-target items were also for the same or similar product types as the target items and were taken from Capitman (1976), Crowley (1979), Exhibitions and Conferences Year Book (1974, 1984), Kompass (1990), Owen’s (1984) Business Directory and Travel Guide, and Ricci and Ferrari (1973a, 1973b). The second pilot study with 15 subjects confirmed that the nontarget brand names were unfamiliar to subjects and were plausible: the pilot study subjects were unable to guess beyond chance frequency which were the real and which the bogus brand names.

(f) Advertisement ratings (including ratings of attitudes towards advertised brands and purchasing intentions): Subjects listened once again to each of the advertisements in turn and were asked to respond to a counterbalanced set of 11 7-point scales measuring attitudes towards advertisement, attitudes towards the brand, intentions to buy the product, and their own estimation of their memory for the advertisements (metamemory). These ratings were based on similar items used in previous research (Homer, 1990; Mackenzie \& Lutz, 1989; Mackenzie, Lutz \& Belch, 1986; Norris \& Colman, 1993; Yi, 1990a). Subjects who had estimated their memory for the advertisement to be very low were not asked to fill in the remaining advertisement ratings scales, because it was considered unreasonable to ask them to provide information on their attitudes towards advertisements that they could not remember. Subjects were also asked if they had heard of the brand name prior to the experiment, to confirm the results of the pilot study that the brand names were unfamiliar to subjects.

\section{Procedure}

Subjects were assigned to treatment conditions quasi-randomly (that is, arbitrarily and virtually randomly within the constraints of timetable availability) and tested in groups of up to twelve. They were told that the research related to psychological aspects of radio listening and that they were about to hear a radio programme lasting about twenty 
minutes, after which they would fill in a few short questionnaires. They were requested to relax and simply listen to the programme. No mention was made of the advertisements. After listening to the programme and advertisements, they were asked to respond to the questionnaires in the order described above. They were not allowed to turn back in order to change or supplement their responses to earlier questionnaires on the basis of information provided in subsequent questionnaires. If subjects were having difficulty remembering the advertisements, they were requested to guess. The completion of the questionnaires was timed: subjects were given two minutes to complete their personal details and the programme ratings, a further four minutes for the completion of the free recall of advertisements questionnaire (b); and two minutes for the remaining three memory questionnaires (c - e). After completing all the questionnaires, the subjects were paid and debriefed. The advertisement ratings questionnaire (f) was untimed.

\section{Scoring of questionnaires}

The 7-point rating scales were each scored from zero (low) to six (high).

The scoring of the recognition measures (c and e above) was straightforward: in each case one point was awarded for a correct choice and zero for an incorrect choice.

Free recall descriptions (b) of the advertisements were marked out of 25 according to lists of 25 salient points created in advance by two independent raters. These covered virtually all the details mentioned in subjects' descriptions. Free recall of product types was scored on a 3-point scale: two marks were awarded for perfectly or virtually correct answers, one mark for substantially correct but insufficiently precise answers (e.g., chocolate bar for ice cream bar), and zero for incorrect answers.

Free recall (b) and cued recall of brand names (d) were scored on 5-point scales: 4 marks were awarded for perfectly correct or virtually correct words (e.g., IXL or Ixel); three marks for almost correct responses but with small mistakes or omissions (e.g., XL for IXL); two marks for answers that were not almost correct but had recognizable elements of the brand's sound (e.g., Excels); one mark for answers with the initial letter correct or the correct number of syllables but no other recognizable elements of brand names sound or appearance (e.g., IFG); and no marks were awarded for completely wrong answers.

The scoring of the free recall and cued recall questionnaires (b and d) was performed by two independent judges using the lists of salient points that had been prepared in advance and the above marking scheme. When there was a discrepancy, the mean of the separate scores was taken. As a check on the reliability of the scoring procedures, correlations between judges' scores were calculated. The correlations were found to be $r=.996(p<.001)$ for free recall and $r=.999(p<.001)$ for cued recall.

The questionnaire measuring perceptions of the radio programmes (f) produced 21 scores pertaining to the 21 individual rating scales and three further scores: an involvement score, an entertainment score, and an enjoyment score based on the empirical definitions of the three terms developed in previous research (Norris \& Colman, 1994b). These are labelled 'global involvement', 'global entertainment', and 'global enjoyment' respectively throughout the Results section of this article. The recall questionnaires (b) and (d) (free recall and cued recall) were combined to form a global recall score. The recognition questionnaires (c) and (e) (recognition of products and brand names) were similarly combined to form a global recognition scale. All the memory scores formed a global memory score for each subject. 


\section{RESULTS}

\section{Correlations between programme ratings and memory for advertisements}

The correlations between subjects' ratings of the programmes and their recall, recognition, and global memory scores for the advertisements were calculated. None of the correlations between subjects' ratings of the radio programmes and memory scores reached significance ( $p>.05$ in each case).

\section{Correlations between programme and advertisement ratings}

The correlations between the programme and advertisement ratings, including attitudes towards the advertised brands and purchasing intentions, are given in Table 1. All the correlations are positive and many are highly significant. Subjects' ratings of the programmes for involvement, entertainment, and enjoyment all correlated positively and significantly with attitudes towards the advertised brand of the product and intentions to buy the product. Correlations between subjects' ratings of the programmes and their attitudes towards the advertisements failed to reach significance.

Table 1. Correlations between programme and advertisement ratings

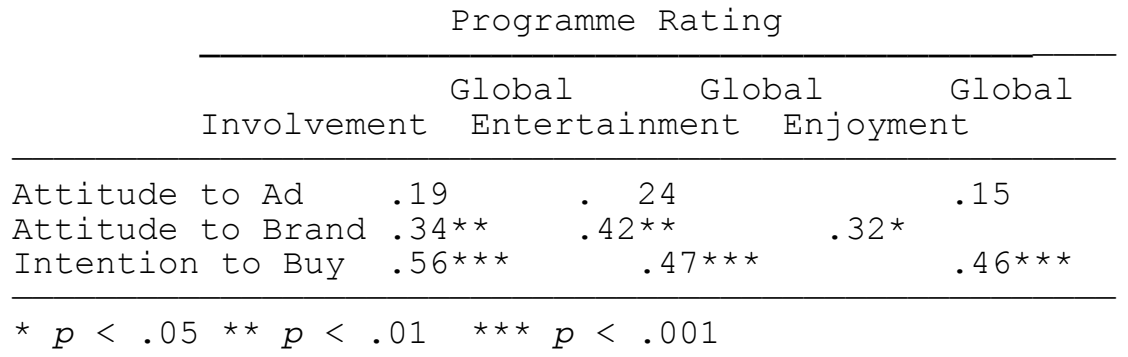

\section{Effect of programme condition on programme ratings}

One-way analyses of variance were performed to evaluate the significance of differences between the mean ratings given to the programmes by the subjects. Mean programme ratings are given in Table 2, together with the results of the analyses of variance.

Table 2. Mean programme ratings across treatment conditions

\begin{tabular}{|c|c|c|c|}
\hline Programme Rating & Phone-In & Chart Show & Nostalgia \\
\hline \multicolumn{4}{|l|}{ Global Ratings } \\
\hline Involvement $\star \star$ & 18.63 & 14.39 & 20.13 \\
\hline 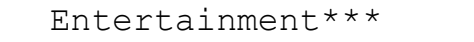 & 6.68 & 7.74 & 10.10 \\
\hline Enjoyment** & 16.94 & 11.45 & 15.00 \\
\hline \multicolumn{4}{|l|}{ Individual Ratings } \\
\hline Involving** & 3.13 & 2.00 & 2.35 \\
\hline Absorbing & 2.26 & 2.26 & 2.65 \\
\hline Stimulating & 2.23 & 2.16 & 2.61 \\
\hline Suspenseful & 1.42 & 1.35 & 1.00 \\
\hline Boring** & 3.90 & 3.45 & 2.77 \\
\hline Interesting* & 2.26 & 2.61 & 3.26 \\
\hline Thought-provoking * * & 2.81 & 1.35 & 2.84 \\
\hline Worth remembering & 1.45 & 1.26 & 2.10 \\
\hline Impact & 2.29 & 2.16 & 2.68 \\
\hline Attention grabbing & 1.97 & 2.13 & 2.65 \\
\hline Challenging & 1.57 & 1.10 & 1.61 \\
\hline Enjoyable*** & 2.29 & 3.03 & 4.00 \\
\hline Exciting & 1.68 & 1.71 & 2.30 \\
\hline Entertaining* & 2.71 & 3.00 & 3.81 \\
\hline Humorous $* \star \star$ & 3.58 & 1.13 & 1.52 \\
\hline
\end{tabular}




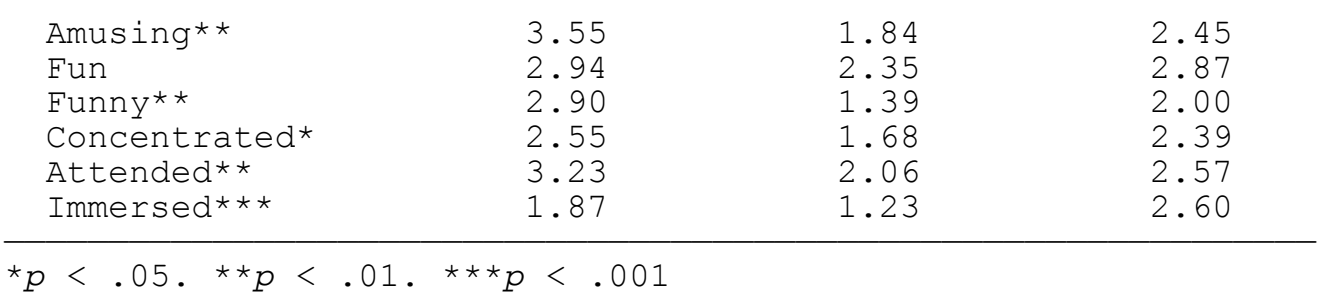

Results of Tukey-HSD tests indicated that the nostalgia programme and the phonein were rated significantly higher than the chart show in global involvement. The phone-in was rated as significantly higher than the chart show in global enjoyment. The nostalgia programme was rated significantly higher than the phone-in and the chart show in global entertainment ratings ( $p<.05$ in each case).

Results of Tukey-HSD tests also revealed that the phone-in was significantly ( $p<$ .05 ) more boring and humorous and significantly less interesting and entertaining than the nostalgia programme. The phone-in was also significantly $(p<.05)$ more involving, thought-provoking, amusing, and funny, than the chart show, and subjects also felt that they attended more and concentrated more during the phone-in compared to the chart show. Subjects felt significantly more immersed in the nostalgia programme than the chart show. Only one item, enjoyment, showed significant differences between all three programmes with the phone-in being significantly less enjoyable than both the nostalgia programme and the chart show, and the chart show being rated significantly less enjoyable than the nostalgia programme ( $p<.05$ in each case).

The analyses of variance revealed no significant differences between the three treatment conditions for subjects' ratings of the programmes as absorbing, stimulating, suspenseful, worth remembering, attention-grabbing, challenging, exciting, fun, and as having impact ( $p>.05$ in all cases).

\section{Effect of programme condition on memory}

Analyses of variance were used to investigate whether the means of the recall, recognition, and global memory scores were significantly different across the three context programmes. Mean recall, recognition, and global memory scores for each of the conditions are given in Table 3, together with the results of the one-way analyses of variance.

Table 3. Recall, recognition, and global memory across treatment conditions

\begin{tabular}{lccc} 
Memory & Phone-In & Chart Show & Nostalgia \\
\hline Recall* & 7.19 & 10.90 & 13.74 \\
Recognition** & 3.23 & 3.61 & 4.73 \\
Global memory* & 10.67 & 14.52 & 18.77 \\
\hline
\end{tabular}

$\star p<.05 . * * p<.01$

The analyses of variance revealed that all three memory scores differed significantly across the radio programmes: recall, $F(2,90)=0.49, p<.05$; recognition, $F(2,88)=4.89, p<.01$; global memory, $F(2,88)=4.92, p<.01)$. In each case a posteriori Tukey-HSD tests indicated that the significance lay between the phone-in and nostalgia programmes. The phone-in produced significantly lower memory scores $(p<.05)$. 
Analyses of variance were used to investigate whether subjects' estimations of their own memory (metamemory) and their advertisement ratings, including their attitudes towards advertised brands and their purchasing intentions, differed significantly across the three radio programmes. The relevant means are shown in Table 4. One-way analyses of variance revealed no significant differences between the means for metamemory, attitudes towards the advertisements and brands, or intentions to buy the products $(p>.05$ in each case).

Table 4. Mean metamemory and advertisement ratings across treatment conditions

\begin{tabular}{lrcc} 
& Phone-in & Chart Show & Nostalgia \\
\hline Metamemory & 10.27 & 11.35 & 12.00 \\
Attitude to advert & 41.00 & 43.32 & 45.70 \\
Attitude to brand & 33.94 & 37.44 & 37.17 \\
Purchasing intention & 25.44 & 22.33 & 30.65 \\
\hline
\end{tabular}

\section{DISCUSSION}

The results of this experiment provide strong evidence for the operation of context effects within the radio medium. The correlations between programme and advertisement ratings - including ratings of advertised brands and purchasing intentions - produced several highly significant and positive results. The more subjects perceived the programmes as enjoyable, entertaining, and involving, the higher were their ratings of the brands and their purchasing intentions. However, the programme ratings did not correlate significantly with attitudes towards the advertisements themselves, although the correlations were positive.

The positive relationship between programme ratings on the one hand and ratings of advertised brands and purchasing intentions on the other may be explained by the operation of some kind of carry-over effect from the programme to the advertisements whereby listeners are more receptive to advertisements while listening to highly rated programmes. Research suggesting that advertisements are more effective within television and print media in comparison to audio-only medium (Furnham, Benson \& Gunter, 1987; Williams, Paul \& Ogilvie, 1957) tend to imply that visual images may evoke more attention than auditory stimuli. With the audio-only radio programmes used in this experiment, subjects had no powerful images to maintain attention, and consequently their attention may have wandered during the transmission. However, when subjects' attention was captured by the more highly rated radio programmes, they were more likely to attend to the advertisements for long enough to form attitudes towards the brands being promoted in the advertisements. It would appear from the results of this study that highly entertaining, involving, and enjoyable programmes are likely to sustain the attention of subjects and to enhance the effectiveness of accompanying advertisements. Thus it could be argued that selective exposure (Thorson, Friestad \& Zhao, 1987; Schumann \& Thorson, 1990) is operating especially strongly within the radio medium.

Alternatively, the positive relationship between program contexts and radio advertisement effectiveness may be explained with reference to the principles of the limited-capacity human information processing system, which has usually been used to explain negative or inverse relationships between contexts and memory for advertisements in research on television viewing (e.g., Norris \& Colman, 1994a). Research findings occasionally suggesting that the medium of radio is superior to 
television in terms of advertisement effectiveness have been interpreted in the light of the fact that television is composed of both auditory and visual stimuli (Klein, 1981; Warshaw, 1978). As a result, according to this interpretation, interference occurs between one sense modality and the other and thus disrupts a recipient's encoding and rehearsal of the television advertisements (Buchholz \& Smith, 1991; Leigh, 1991; Warshaw, 1978). Thus it is possible that the context of a radio programme produces less interference for the processing of the accompanying advertisements because it uses only one sense modality, namely audition, and this could be why a positive relationship is observed between programme ratings and advertisement effectiveness.

Compared to the above results, the correlations between programme ratings and memory for the advertisements were all non-significant, providing no evidence that programme environments influence memory for embedded advertisements within the radio medium. Thus the findings relating to memory for advertisements on the one hand and ratings of advertised brands and purchasing intentions on the other have turned out to be quite different. It is not clear why programme-induced involvement, entertainment, and enjoyment are significantly related to attitudes towards the brands and purchasing intentions but not to memory for those advertisements. These results tend to suggest that memory for advertisements are affected in a different way by program contexts than are attitudes towards advertised brands and purchasing intentions. It is possible that while the effects of highly involving, entertaining, and enjoyable programmes may carry over to sustain listeners' attention during the advertisements for long enough to enhance their attitudes towards brands and their purchasing intentions, they are not sufficient to form durable memory traces, and thus programmes differing in entertainment, enjoyment, and involvement properties produce differential effects on attitudes towards brands and purchasing intentions but not on memory for advertisements (Norris \& Colman, 1993).

Analyses of variance revealed highly significant differences across the three radio programmes in memory for the advertisements. Measures of recall, recognition, and global memory for the advertisements all yielded significantly lower scores among subjects who heard the phone-in programme than those who heard the nostalgia programme. These memory results coincided with some interesting findings regarding subjects' ratings of the respective radio programmes. The results of the Tukey-HSD tests revealed that the phone-in was significantly more enjoyable (measured with global ratings), involving (global and individual ratings), thought-provoking, amusing, funny, and elicited more concentration and attention than the chart show. Results also showed that phone-in was rated as significantly less interesting, entertaining (global and individual ratings), and enjoyable, but significantly more boring and humorous than the nostalgia programme. Some of the programme ratings may appear paradoxical — for example, the phone-in was rated as both boring and humorous - but the cluster analysis reported in Norris and Colman (1994b) showed that the perceived entertainment value, enjoyableness, and involvement of programmes (in that case, television programmes) are largely independent of one another (see also Norris \& Colman, 1993, 1994a). Taken together, the results regarding memory and programme ratings imply that advertisements were more effective in terms of memory when embedded in programmes that were rated significantly more entertaining, enjoyable, interesting, and less boring and humorous. However, this inference is tentative because the analyses of variance cannot test the relationship between programme ratings and memory directly. Any differences between the three programmes in terms of advertisement effectiveness may be due to numerous other programme factors apart 
from the specific programme ratings that were measured in this experiment. A programme-only control condition could obviously not be included in the experimental design, because the key dependent variables were measures of memory and ratings of advertisements, and an advertisements-only condition was not included for reasons of ecological validity, because in naturally occurring situations people do not listen to radio advertisements in the absence of any programming context.

The most important finding of this study was that cognitive processing of advertisements was subject to substantial context effects operating in the radio medium. In particular, memory scores for the advertisements and programme ratings were found to differ significantly between the three programme contexts. The phone-in programme produced significantly lower memory scores than the nostalgia radio programme. In addition, programme-induced involvement, entertainment, and enjoyment were positively related to attitudes towards brands and purchasing intentions. It would be useful to investigate the effects of context on exposure to both novel and familiar radio advertisements using a naturalistic research environment, and the replicability of the findings described in this article needs to be checked with a different range of context material.

\section{REFERENCES}

Abernethy, A. M. (1991). Differences between advertising and program exposure for car radio listening. Journal of Advertising Research, 31(2), 33-42.

Axelrod, J. N. (1963). Induced moods and attitudes toward products. Journal of Advertising Research, 3(2), 19-24.

Bryant, J. \& Comisky, P. W. (1978). The effect of positioning a message within differentially cognitively involving portions of a television segment on recall of the message. Human Communication Research, 5(1), 63-75.

Barclay, W. D., Doub, R. M., \& McMurtrey, L. T. (1965). Recall of TV commercials by time and program slot. Journal of Advertising Research, 5(2), 41-47.

Buchholz, L. M., \& Smith, R. E. (1991). The role of consumer involvement in determining cognitive response to broadcast advertising. Journal of Advertising, 20(1), 4-17.

Capitman, B. B. (1976). American trade designs: A survey with 732 marks, logos, and corporate identity symbols. New York: Dover.

Clancy, K. J., \& Kweskin, D. M. (1971). TV commercial recall correlates. Journal of Advertising Research, 2, 18-20.

Crowley, E. T. (1979). Trade names dictionary (2nd Edition). Detroit, MI: Gale Research Company.

Duncan, C. P., \& Nelson, J. E. (1985). Effects of humor in a radio advertising experiment. Journal of Advertising, 14(2), 33-40.

Exhibitions and Conferences Year Book. (1974). London: York Publishing.

Exhibitions and Conferences Year Book. (1984). London: York Publishing.

Furnham, A., Benson, I., \& Gunter, B. (1987). Memory for television commercials as a function of the channel of communication. Social Behaviour, 2, 105-112.

Gelb, B. D., \& Zinkhan, G. M. (1985). The effect of repetition on humor in a radio advertising study. Journal of Advertising, 14(4), 13-20.

Gelb, B. D., \& Zinkhan, G. M. (1986). Humor and advertising effectiveness after repeated exposures to a radio commercial. Journal of Advertising, 15(2), 15-20.

Heflin, D. T. A., \& Haygood, R. C. (1985). Effects of scheduling on retention of advertising messages. Journal of Advertising, 14(2), 41-47, 64. 
Homer, P. M. (1990). The mediating role of attitude toward the ad: Some Additional evidence. Journal of Marketing Research, 27(1), 78-86.

Horn, M. I., \& McEwen, W. J. (1977). The effect of program context on commercial performance. Journal of Advertising, 11, 23-27.

Kamins, M. A., Marks, L. J., \& Skinner, D. (1991). Television commercial evaluation in the context of program-induced mood: Congruency versus consistency effects. Journal of Advertising, 20(2), 1-14.

Kennedy, J. R. (1971). How program environment affects TV commercials. Journal of Advertising Research, 11(1), 33-38.

Klein, G. (1981). Relative advertising channel effectiveness: A test of learning vs. involvement orientations. Current Issues and Research in Advertising, 4, 71-84.

Kompass. (1990). Register of industry and commerce of Canada: Products and Services (13th ed.). Toronto: Kompass.

Krugman, H. E. (1983). Television program interest and commercial interruption: Are commercials on interesting programs less effective? Journal of Advertising Research, 23(1), 21-23.

Leigh, J. H. (1991). Information processing differences among broadcast media: Review and suggestions for research. Journal of Advertising, 20(2), 71-75.

Lloyd, D. W., \& Clancy K. J. (1991). CPMs versus CPMIs: Implications for media planning. Journal of Advertising Research, 31(4), 34-44.

MacKenzie, S. B., \& Lutz, R. J. (1989). An empirical examination of the structural antecedents of attitude toward the ad in and advertising pretesting context. Journal of Marketing, 53, 48-65.

MacKenzie, S. B., Lutz, R. J., \& Belch, G. E. (1986). The role of attitude toward the ad as a mediator of advertising effectiveness: A test of competing explanations. Journal of Marketing Research, 23, 130- 43.

Murphy, J. H., Cunningham, I. C. M., \& Wilcox, G. B. (1979). The impact of program environment on recall of humorous television commercials. Journal of Advertising, 8, 17-21.

Norris, C. E., \& Colman, A. M. (1992). Context effects on recall and recognition of magazine advertisements. Journal of Advertising, 21(3), 37-46.

Norris, C. E., \& Colman, A. M. (1993). Context effects on memory for television advertisements. Social Behavior and Personality, 21, 279-296.

Norris, C. E., \& Colman, A. M. (1994a). Effects of entertainment and enjoyment of television programs on perception and memory of advertisements. Social Behavior and Personality, 22, 365-367.

Norris, C. E., \& Colman, A. M. (1994b). Putting ads in context: How television programmes affect viewers' reactions to ads. Admap, 29(1), Issue 336, 39-42.

Owen's business directory and travel guide: Middle east, Mediterranean, Africa, and Indian Ocean, Asia. (1984). Oxford: Owen's Publications.

Park, C. W., \& McClung, G. W. (1986). The effect of TV program involvement on involvement with commercials. Advances in Consumer Research, 13, 544-548.

Ricci, F. M., \& Ferrari, C. (Eds.). (1973a). Top symbols and trademarks of the world: 1 United States. Milan: Deco Press.

Ricci, F. M., \& Ferrari, C. (Eds.). (1973b). Top symbols and trademarks of the world: 2 United States, Part 2, Canada. Milan: Deco Press.

Schumann, D. W. (1986). Program impact on attitude toward TV commercials. In J. Seagert (Ed.), Proceedings of the Division of Consumer Psychology (pp. 67-73). Washington, DC: American Psychological Association. 
Schumann, D. W., \& Thorson, E. (1990). The influence of viewing context on commercial effectiveness: A selection processing model. In J. H. Leigh \& C. R. Martin (Eds.), Current issues and research in advertising, 12(1), 1-24.

Schwerin, H. S., \& Newell, H. H. (1981). Persuasion in marketing. New York, Wiley.

Soldow, G. F., \& Principe, V. (1981). Response to commercials as a function of program context. Journal of Advertising Research, 21(2), 59-65.

Thorson, E., Friestad, M., \& Zhao, X. (1987). Attention to program context in a natural viewing environment: Effects on memory and attitudes toward commercials. Paper presented at the Association for Consumer Research, Boston, October.

Thorson, E., \& Reeves, B. (1986). Effects of over-time measures of viewer liking and activity during programs and commercials on memory for commercials. In R. Lutz (Ed.) Advances in consumer research (Vol. 13). Provo, UT: Association for Consumer Research.

Thorson, E., Reeves, B., \& Schleuder, J. (1985). Message complexity and attention to television. Communication Research, 12, 427-454.

Warshaw, P. R. (1978). Application of selective attention theory to television advertising displays. Journal of Applied Psychology, 63, 366-372.

Williams, D., Paul, J., \& Ogilvie, J. (1957). The mass media, learning and retention. Canadian Journal of Psychology, 11, 159-163.

Yi, Y. (1990a). Cognitive and affective priming effects of the context for print advertisements. Journal of Advertising, 19(2), 40-48.

Yi, Y. (1990b). The effects of contextual priming in print advertisements. Journal of Consumer Research, 17, 215-222.

\section{AUTHOR NOTES}

We are grateful for the assistance of Dr Chris Nicklin in scoring the questionnaires. Requests for reprints should be sent to Dr Claire E. Norris, Department of Human Communication, De Montfort University, Scraptoft Campus, Leicester LE7 9SU, England. 